\title{
Le récit du rapt de Perséphone: interprétations d'un "mythe" et performance rituelle
}

\author{
CLAUDE CALAME \\ Université de Lausanne
}

\begin{abstract}
RESUMO: A narrativa do rapto de Perséfone nos oferece um exemplo ideal para propor, ao mesmo tempo, uma crítica à moderna noção de mito e um percurso pelas diversas interpretações que tal narrativa, considerada como mito, pode suscitar. Somente o estudo de uma versão poética particular, recolocada em suas circunstâncias rituais de enunciação por meio de uma abordagem simultaneamente antropológica e semio-narrativa, é capaz de dar conta dos valores e da função próprios de um mito.
\end{abstract}

PALAVRAS-CHAVE: Poética; mito grego; Deméter e Perséfone; hermenêutica.

En guise de prélude à une étude d'un récit hellène qui dès l'Antiquité a sollicité l'imagination des herméneutes, on commmencera par une affirmation quelque peu péremptoire et provocatrice sur le texte narratif qu' on a coutume d'appeler mythe. On postulera en effet que, désormais entrée dans le savoir partagé de notre culture académique, la notion anthropologique du "mythe" n'a de pertinence pour la Grèce antique qu'à la condition de séparer les récits poétiques des Hellènes de toute forme littéraire, et de les réduire à quelques noms propres animant une intrigue. C'est en fait avec les manuels de mythographie que les récits grecs deviennent, au-delà de leurs multiples formes de mise en discours, de simples squelettes; ils sont alors métamorphosés en des "mythes" auxquels on croit pouvoir accorder, en raison de leur réduction à une forme schématique, une existence transcendante. C'est ainsi également que, pour nos contemporains mythologues de l'Antiquité, le "mythe" peut devenir un simple "récit traditionnel", doublé d'une référence sociale'. Cette forme d'écriture savante, à travers des collections érudites de résumés purement narratifs, a d'ailleurs permis au patrimoine légendaire des Grecs de parvenir jusqu'à la Renaissance, avant qu'on ne commence à en relire la réalisation poétique dans l'Iliade et l'Odyssée ou dans les tragédies d'Euripide, mais aussi dans les drames de Sénèque. 


\section{La notion moderne de "mythe"}

L'établissement de cette tradition narrative dans une élaboration secondaire, savante et académique, explique en partie l'évolution connue dès le XVIII ${ }^{e}$ siècle par les catégories du "mythe" et de la "mythologie". En ce qui concerne la tradition française, ce n'est à vrai dire qu'à partir du début du XIX ${ }^{\mathrm{e}}$ siècle que dans les dictionnaires encyclopédiques, l'entrée mythe se substitue à celle intitulée fable. Dans l'Encyclopédie de d'Alembert et Diderot, la fable est ce "nom collectif sans pluriel, qui renferme l'histoire théologique, l'histoire fabuleuse, l'histoire poétique, et pour le dire en un mot, toutes les fables de la théologie payenne"; une théologie qui se réfère d'ailleurs essentiellement à l'Antiquité gréco-romaine et dont les "fables" figurent sous la rubrique myth(ologie). Tant il est vrai que, si mythe est absent de l'Encylopédie, ce terme est en revanche couramment utilisé dans les ouvrages du même type qui lui succéderont au siècle suivant. Avec l'introduction de mythus Christian Gottlob Heyne fait donc figure de précurseur².

On remarque ainsi qu'à la faveur de la renomination de la fabula en mythus, le concept de mythe subit dans la pensée anthropologique en formation trois métamorphoses essentielles. Tout d'abord considérés comme des expressions et manifestations préphilosophiques propres aux peuples sauvages, les mythes sont situés à l'aurore d'une histoire linéaire qui ne peut conduire qu'aux progrès éclairés et à l'achèvement métaphysique de la civilisation occidentale. Le jeu de mot implicite sur la notion de "primitif" est l'emblème d'une projection dans la dimension historique du rôle attribué au mythe dans les cultures spatialement les plus distantes. L'Antiquité vient alors tout naturellement prendre sa place, avec ses légendes puis ses philosophes, au début de la ligne évolutive censée permettre à la culture européenne de se dégager de l'obscurité du mûthos et d'accomplir les pas décisifs vers la rationalité attribuée au lógos. A l'occasion de cette projection d'une image spatiale de la culture des hommes sur une représentation temporelle, la notion de mythe connaît une seconde transformation. Par la réduction progressive de la pluralité des récits entendus sous ce terme à l'unicité d'un concept, le mythe semble perdre son aspect narratif pour devenir l'expression d'un mode particulier de la pensée humaine. Il correspondrait ainsi à la forme de raison spécifique des cultures primitives.

Le statut du mythe comme indice d'un état premier de civilisation provoque une ontologisation qui, aux yeux des anthropologues puis dans le savoir partagé de l'intelligentsia occidentale, lui confère une valeur universelle. Né en tant que l'une de ces catégories du "niveau de base" qui par son caractère semi-empirique est un instrument de connaissance, le mythe est passé du statut de notion opératoire à celui d'une réalité transcendantale. Mais à la faveur de ce glissement lexical et conceptuel, le mythe est aussi devenu mythologie, entendue non seulement comme la collection des récits fabuleux d'une culture particulière, mais aussi comme la science que requiert leur interprétation. Mythe, mode de pensée; mais, à la faveur d'une troisième métamorphose, le mythe transformé en substance suscite encore la science du mythe.

De ces trois transformations autant l'intitulé que le but assigné aux Mythologiques par leur auteur Claude Lévi-Strauss portent la marque. Etude comparative transformationnelle et formelle - et donc mythologie - d'un corpus de récits en général réduits à la textualité d'une intrigue pour découvrir la logique qui les traverse. Cette "logique du concret", qui se 
nourrit du contexte ethnographique, est aussi l'indice d'une pensée mythique et de son universelle diffusion. Pour Lévi-Strauss, "les schèmes mythiques offrent au plus au point le caractère d'objets absolus qui, s'ils ne subissaient des influences externes, ne perdraient ni n'acquerraient de parties. Il en résulte que, quand le schème subit une transformation, celleci affecte solidairement tous ses aspects (...). Ce qui importe, c'est que l'esprit humain, sans égard pour l'identité de ses messages occasionnels, y manifeste une structure de mieux en mieux intelligible à mesure que progresse la démarche doublement réflexive de deux pensées agissant l'une sur l'autre..."

Tout en restituant le Grand Partage qui doit nous séparer des autres par une série de traits distinctifs érigés en substances (primitif/évolué, oral/écrit, froid/chaud, nature/culture, mythe/raison, etc.), l'idée d'une logique transformationnelle des mythes érigée en mode universel de la "pensée sauvage" contribue à réduire singulièrement autant extraordinaire richesse sémantique de ces manifestations discursives que l'éventail des fonctions sociales et symboliques que peuvent assumer des récits toujours attachés à une mise en discours et à des conditions d'énonciation singulières.

\section{Le récit du rapt de Perséphone: versions antiques}

La mythologie transformée à la fois en mytho-logique et en mytho-logie a au moins eu cet effet positif de susciter une effervescence interprétative tout à fait remarquable. En accord avec les différents paradigmes épistémologiques qui ont contribué à leur apparition, les moments marquants de cette herméneutique des mythes forment désormais une succession et donc une histoire tout à fait remarquables ${ }^{4}$. En ce qui concerne la Grèce antique, on peut retracer certaines des étapes décisives de ce développement en évoquant quelquesunes des interprétations successivement suscitées par ce qui est devenu le "mythe de Déméter et Coré" ou le "mythe du rapt de Peséphone". L'histoire du rapt de Perséphone et de la recherche de la fille par sa mère présente l'intérêt particulier d'avoir suscité une certaine curiosité interprétative déjà dans l'Antiquité. En se fondant sur différentes manifestations littéraires dans le travail de collection du savoir encyclopédique de la tradition mythographique que l'on a mentionnée, historiographes et mythographes hellènes n'ont pas manqué de donner de la célèbre histoire un résumé qui la réduit au déroulement de son intrigue.

On peut suivre, par exemple, le texte de la Bibliothèque attribuée à Apollodore, on retrouve dans cet authentique ouvrage de mythographie l'amour que Pluton ressent pour Perséphone et l'aide de Zeus dont bénéficie le dieu des Enfers pour enlever la jeune fille en secret; l'arrivée de Déméter à Eleusis, l'hospitalité offerte par le roi Céleus et le rire que Iambé parvient à susciter auprès de la déesse en deuil de sa fille; la tentative d'immortalisation de Démophoon, le jeune fils de Céleus et de Métanire, et le don à Triptolème, le fils aîné des souverains d'Eleusis, du chariot entraîné par des dragons ailés qui permettra au jeune héros d'ensemencer la terre entière; enfin le retour de Perséphone sur l'ordre de Zeus à Pluton qui, par la ruse du pépin de grenade, contraint néanmoins sa jeune épouse à passer un tiers de l'année dans les profondeurs de l'Hadès alors qu'elle résidera parmi les dieux pour le reste de l'an. Le résumé donné par la Bibliothèque posthellénistique suit en fait la version du récit 
que développe à l'époque archaïque l'Hymne homérique à Déméter; à cette exception près qu'elle introduit le célèbre épisode de Triptolème; dans l'iconographie comme dans les textes, cet épisode absent de l'Hymne fait de l'histoire du rapt de Perséphone un récit centré sur Athènes et sur une Eleusis désormais intégrée au territoire de l'Attique. C'est en effet en tant que héros culturel spécifiquement athénien que Triptolème reçoit de Déméter le don de la céréaliculture et qu'il devient ainsi le bienfaiteur de l'humanités

Mais n'anticipons pas. En effet, dès l'époque classique, il se trouve des gens de lettres non seulement pour mettre en scène par différents moyens sémiotiques et pour réorienter le sens du récit du rapt de Coré, mais aussi pour réfléchir à sa signification. Ainsi en va-t-il par exemple du rhéteur et philosophe Isocrate. Dans le discours d'apparat qu'il a rédigé à la louange de sa propre cité, l'orateur athénien en vient à s'interroger non seulement sur l'origine des biens et des dons dont Athènes a disposé dès le début (ex arkhês), mais aussi sur les bienfaits qui, dès le commencement de son histoire (ap'arkhês), en ont été la conséquence pour les autres. Question de l'origine (arkhé) et question de la cause ou de la responsabilité (aitia) sont étroitement mêlées dans une interrogation qui englobe toute l'humanité dans toute l'étendue du temps, pour une tradition mémoriale (legoménas kai mnemoneuoménas) qui lie le présent au passé le plus reculé (kai pálai kaì nûn $)^{6}$.

Au début donc (prôton toínun), selon Isocrate, l'arrivée de Déméter en Attique errant à la recherche de Coré. En reconnaissance pour l'accueil reçu, Déméter accorde aux ancêtres des Athéniens un double don: les fruits de la terre qui leur permirent d'abandonner le mode de la vie sauvage, et les mystères qui continuent à entretenir pour les mortels l'espoir d'une fin et d'un au-delà plus doux. De ces dons divins, les Athéniens firent bénéficier tous les autres hommes. Sans doute le récit (lógos, et non pas mûthos) peut-il recevoir la qualification de "fictionnel" (muthódes). Il convient néanmoins non seulement de le raconter, maintenant également (kaì nûn), dans le temps de l'énonciation; mais les raisons sont aussi nombreuses pour lui accorder créance. La longue tradition qu'impliquent des dits aussi anciens (arkhaîa) appelle la confiance. A cela s'ajoutent les indices (semê̂a) que constituent les prémisses du blé nouveau envoyés chaque année à Athènes par différentes cités en souvenir de l'ancien bienfait (hupómnema tês palaiâs euergesías) ainsi que l'ordre donné par la Pythie de Delphes aux autres cités d'en faire autant. Ce geste rituel, dans sa diffusion panhellénique, est le témoignage même (summarturê̂) de l'accord entre la pratique actuelle (nûn) et la tradition d'autrefois (pálai). L'ancienneté même des actes entrés dans la mémoire communautaire finit donc par fournir la garantie de leur historicité; cette vérité historique s'appuie autant sur la relation que ces actions légendaires entretiennent avec la pratique (rituelle) du présent que sur leur utilité pour la conduite d'une vie heureuse.

Dans le discours d'Isocrate, le récit du rapt de Perséphone entretient une relation étroite avec le présent; relation discursive dessinée soit par la tradition, soit par le continuum du comput chronologique; mais relation sociale aussi, établie dans les deux cas par la pratique rituelle et par ses effets sur la conduite de la vie des hommes dans le présent de leur action communautaire. Même s'il est l'objet d'une réflexion rhétorique ou historiographique, le mûthos est toujours actif dans le présent; même si son insertion dans un autre type de lógos implique qu'il soit résumé, c'est l'ensemble de ses valeurs qu'actualise son rapport avec la pratique sociale. La transformation de ce discours figuré en un discours plus abs- 
trait, de nature interprétatif, ne s'accomplira que dans la réflexion spéculative des philosophes ou avec le changement de paradigme culturel qui se dessine à l'époque de l'Antiquité tardive. Qu'elle se réalise dans l'espace, dans le temps ou par des procédures intellectuelles, l'activité interprétative requiert en fait la distance qu'exige la traduction dans ce qu'on appelera un autre régime d'intelligibilité. Pour l'orateur du IVe siècle, l'histoire du rapt de Perséphone est encore une version narrative pleinement active.

\section{Interprétations indigènes}

Dans le De natura deorum, Cicéron porte son intérêt sur la nomination des dieux du système polythéiste gréco-romain, comme le Platon du Cratyle. En bon rhéteur pétri de culture grecque, il est sensible aux jeux étymoligisants qui permettent de déduire la nature ou l'essence d'un être de la signification qu'indique sa dénomination. Dans le développement de leur conception du cosmos, les Stoïciens ont fait un large usage des possibilités sémantiques offertes par les noms des dieux du panthéon, traduisant ainsi en des entités plus abstraites les figures anthropomorphes de la théologie traditionnelle. Pour l'orateur latin, cette lecture allégorique des noms des dieux grecs et romains correspond à une transformation de "fables impies" (impiae fabulae) en une théorie physique (physica ratio) qui ne manque pas d'élégance. A en croire Cicéron donc, Zénon ainsi que, à sa suite, Cléanthe et Chrysippe divisent le cosmos entre ciel, mer et terre. Le règne terrestre, avec sa puissance naturelle, avec les fruits qui tombent en son sein et qui en resurgissent, est attribué à Dis le père. Cette figure paternelle est rapprochée avec d'autant plus de facilité de la richesse désignée en latin par dives que son équivalent grec Pluton (Ploutôn) réfère étymologiquement à la même abondance de biens. Dans ce contexte de procédures étymologico-allégoriques, Proserpine, appelée par les Grecs Persephóne, devient le grain caché puis recherché par la mère, elle-même l'objet d'un double jeu étymologisant. Le nom latin de la déesse, Ceres, est censé évoquer "l'entretien" ou "l'administration" (geres de gerere) des fruits de la terre tandis que son correspondant grec doit permettre, également par la mutation d'une seule lettre, de faire de Déméter la terre mère (gê méter)?

Coré, figure anthropomorphe et théologique non pas simplement du blé ou du printemps, mais du processus attaché à la céréaliculture. C'est bien par la projection implicite de l'histoire de son rapt par Pluton et de son retour auprès de Déméter sur le cycle de l'année, à partager désormais entre l'Hadès et l'Olympe, que le "mythe" de Perséphone peut devenir l'allégorie de la croissance du blé dans l'alternance des saisons. Coré non pas comme simple figure allégorique, mais comme protagoniste d'un processus dynamique: les jeux de mots étymologisants sur les noms des autres protagonistes de l'action, Pluton et Déméter, en confirment les valeurs attachées à la production des fruits de la terre. Conduisant dans le cas de l'enlèvement de Coré du récit figuratif à l'alternance naturelle des saisons et à la procédure socialisée de la culture des céréales, la démarche interprétative d'abstraction se montre donc influente dès l'Antiquité. Cette perspective herméneutique est possible dès qu'un point de vue de philosophes permet de prendre une certaine distance visà-vis de traditions légendaires qui, à leurs yeux, ne sont plus attachées à une pratique cultuelle particulière. Les Stoïciens ne se font forcément initier aux Mystères d'Eleusis... 


\section{Herméneutiques modernes}

C'est précisément en s'appuyant sur l'autorité d'un historien ancien qu'au début du XVIII ${ }^{e}$ siècle, le père jésuite Jean-François Lafitau parvient à comparer les "Initiations de Bacchus et de Cérès" avec les "Orgies d'Isis et d'Osiris" pour les classer dans la même grande catégorie des "Mystères". On y retrouve, pêle-mêle, les mystères de Bacchus et de la Mère des dieux de Thrace, ceux de Vénus et d'Adonis à Chypre et en Phénicie ou ceux d'Atys et de Cybèle en Phrygie. A partir d'un décor originaire marqué par "la même Divinité et le même fonds de Religion", il s'agit d'offrir à Monseigneur le Duc d'Orléans "une peinture des Moeurs des Peuples du nouveau-Monde". Et le père de la Compagnie de Jésus d'ajouter: "Ces Moeurs, et le parallèle que j'en fais avec celles des premiers temps, ne présentent que des dehors sauvages et des Coûtumes barbares, qui sont bien éloignés de la politesse de nôtre siècle et de nôtre Nation". Au sein du grand panorama d'anthropologie comparative et évolutive brossé par Lafitau, Coré-Proserpine trouve sa place, à la fois en tant que jeune fille et comme mère par Jupiter de Bacchus liber. Elle représente dès lors une image de la Vierge des Chrétiens, mère du Fils Libérateur. Elle est donc l'une de ces figures de la "Théologie des premiers temps" qui est aussi, à travers la "fable allégorique", celle des Iroquois ou celle des Caraïbes contemporains ${ }^{8}$. Mais, dans l'entreprise comparative du Siècle des Lumières, Coré va rapidement être appelée à rejoindre les champs de blé.

Parallèlement au Père Lafitau, Giambattista Vico voit dans la théologie grecque une première "sagesse poétique", une métaphysique non pas raisonnée et abstraite, mais analogue à celle ressentie et imaginée par les premiers hommes delle nazioni gentili. A l'aurore de la civilisation, les sages parmi ces premiers rustres sont des poètes au sens grec du terme, c'est-à-dire des créateurs. Ces poètes théologiens sont aussi les auteurs d'une "physique poétique" qui attribue aux éléments et aux forces de la nature la figure et le nom de dieux, parvenant ainsi parfois à la constitution d'un vrai canone mitologico. Dans cette perspective allégorique reprise à l'Antiquité et probablement à la suite des Stoïciens cités par Cicéron, Proserpine est enlevée dans les Enfers par "Dite", le Pluton des Grecs, qui est le représentant par étymologie interposée des richesses de la terre. Mais dans ce processus d'explication allégorique, Proserpine est identifiée avec Cérès pour devenir le symbole des grains de blé que l'on enfouit dans les sillons assimilés aux profondeurs de l'Hadès. Cérès est aussi la maîtresse des règles de l'agriculture, fondement d'un premier droit foncier de l'âge des héros 9 .

Mais pour que le comparatisme soit institué en méthode en ce qui concerne la mythologie grecque, il faudra attendre non pas le célèbre Karl Otfried Müller, mais l'autre Müller, le linguiste émigré à Oxford, Friedrich Max de son prénom. Dans sa neuvième Lecture on the Science of Language, le distingué historien de la langue ne se contente pas de montrer "the crudities and absurdities" de certains épisodes de ce qu'il appelle The Mythology of the Greeks (à nouveau la mythologie comme corpus de mythes et non plus comme approche scientifique!). Ce faisant, moins qu'à "dénoncer un scandale" selon la formule un peu tapageuse un temps à la mode à Paris, le second Müller se met du côté des philosophes grecs eux-mêmes, sensibles à l'amoralité, sinon à la monstruosité de certains des personnages et de certaines des actions mis en scène dans les récits de leurs poètes. Car si les mythes, dans leurs incohérences, peuvent bien être présentés comme des perversions linguistiques, ils 
méritent néanmoins notre attention érudite; ils requièrent interprétation. Au-delà des explications éthiques, physiques ou historiques formulées par les Anciens eux-mêmes, le linguiste propose l'approche nouvelle que constitue la philologie comparée. Dans le cadre du comparatisme indo-européen, c'est à nouveau l'étymologie qui est mise au service de l'herméneutique. A partir de l'analogie phonétique qui associe le grec theós et le latin deus au sanscrit deva, on abandonne les jeux étymologisants proposés par Hérodote, qui dérive theós de tithérai, disposer, ou par Platon qui voit l'origine du même mot dans la course (théein) du soleil et de la lune, dieux primordiaux. A ce jeu nouveau, Déméter est associée à son avatar Déô qui dériverait du Dyâvâ védique; Déméter est alors Dyâvâ mâtar, à la fois Aube et Mère, correspondant de Dyausch pitar, Ciel et Père, en grec Zeus ${ }^{10}$.

Ainsi insérée dans le paradigme de la langue originaire des Indo-européens reconstruite par la linguistique historique du XIX ${ }^{\mathrm{e}}$ siècle, Déméter sera appelée, pour une brève période, à abandonner la terre fertilisée et la céréaliculture pour ne régner que sur le ciel, en tant que mère. Mais la grande entreprise comparative du Rameau d'Or ne va pas tarder à faire regagner à la déesse les champs labourés. Pour James Frazer, le scénario est connu, défini en substance tour à tour par l'histoire syrienne d'Aphrodite-Astarté et d'Adonis, par le mythe phénicien de Cybèle et d'Attis ainsi que par le récit égyptien d'Isis et d'Osiris. En passant implicitement du groupe des langues indo-européennes au paradigme sémitique, le mythe de Déméter et Perséphone ne ferait que répéter le scénario qui fonderait également mythes et rites dionysiaques: la mort et la résurrection de la végétation". Tel serait donc le "noyau originaire" (the original kernel) du mythe tel que le raconterait déjà l'Hymne homérique à Déméter avec l'équivalence suivante: "une déesse porte le deuil d'un ou d'une aimée qui personnifie la végétation, plus spécifiquement le blé, qui meurt en hiver et renaît au printemps".

Personnification est donc le maître-mot qui permet de passer de la manifestation narrative et figurative à son noyau de sens. Dans le cas précis de Déméter et de sa fille Perséphone, la relation énigmatique entre niveau de surface et niveau profond est d'un déchiffrement aisé. La fille comme la mère sont à lire comme des personnifications du grain; la première parce que, selon les versions du mythe, elle passe chaque année trois à six mois dans les profondeurs de la terre qui sont celles où sont enfouies les semences pendant l'hiver, et parce que son retour au printemps coïncide avec la renaissance des jeunes pousses du blé et avec celle de la végétation en général; la seconde dans la mesure où, par analogie avec sa fille personnifiant le jeune grain de l'année nouvelle, elle pourrait représenter moins la terre, comme le pensaient les Anciens, que le grain récolté l'année précédente et ensemencé à l'automne. Le récit du rapt de Perséphone dans l'Hadès, de sa recherche par Déméter et de son retour à la surface terrestre ne ferait donc que redire le cycle saisonnier de la végétation et de la culture des céréales compris comme alternance entre mort et résurrection. Avec leur promesse d'une immortalité bienheureuse, les mystères d'Eleusis ne feraient quant à eux que répéter rituellement ce cycle; en compagnie de Déméter comprise comme Corn Mother et de Perséphone représentant la Corn Maiden, il nous conduiraits "de la tristesse mélancolique et de la décomposition de l'automne à la fraîcheur, la lumière et la floraison verdoyante du printemps" 12 . Ainsi, par abstractions successives à partir du même scénario, on est passé de la relation entre deux divinités anthropomorphes au cycle de la croissance végétale pour aboutir, avec le rituel, au fondement de la destinée de l'homme, partagée entre la mortalité et différentes formes de renaissance. 
C'est au nom d'un retour très protestant à la nature et à la réalité des travaux agricoles que l'historien des religions suédois Martin P. Nilsson a été amené à préciser, avec d'autres collègues, la chronologie calendaire supposée être fondée sur le cycle naturel des saisons. Si une connaissance empirique des conditions climatiques et du rythme des travaux agricoles en Grèce empêche de voir en Coré la Kornmädchen qui, enlevée au temps des fleurs, passerait les quatre mois d'hiver sous terre et le reste de l'année avec sa mère à la surface, alors le cycle de Déméter et Perséphone, considérées toutes deux comme déesses des semailles, doit être inversé: la séjour de Coré dans l'Hadès ne peut plus que correspondre aux quatre mois de l'été grec où les champs sont secs et nus ${ }^{13}$. Dans ce scénario au rythme revisité, désormais proche du Naturleben, Coré représenterait les semences déposées dans les pithoi en juin, ceci en accord avec le moment de son rapt et de sa katagogé que l'on célèbre en Sicile à l'époque de la récolte; et, en conséquence, au moment de l'ánodos de la jeune femme correspondrait la période des semailles, marquée par la célébration éleusinienne. A cette dimension synchronique de l'interprétation naturalisante s'ajoute naturellement la composante diachronique d'une herméneutique également historicisante. Par étymologie interposée, mais aussi grâce à la place attribuée au serpent à la fois dans le complexe mythico-rituel éleusinien et dans la religion minoenne, Perséphone est métamorphosée en déesse de la végétation préhellénique. Cette origine prestigieuse ne fait que confirmer le statut de Coré en tant que Kornmädchen, à côté de Déméter la Kornmutter! Désormais élevée au rang de paradigme herméneutique, la relation ontologique des deux déesses avec le blé et sa croissance connaîtra pendant tout le $\mathrm{XX}^{\mathrm{e}}$ siècle une remarquable fortune puisque même pour le prudent commentateur anglais de l'Hymne homérique à Déméter, Fille et Mère, à l'origine confondues, restent attachées à la jeune moisson, puis au blé mûr. Dans ce contexte interprétatif est également envisagée une transposition de ce processus végétal sur la vie des hommes divisée en croissance attachée à la jeunesse et maturité de l'âge adulte ${ }^{14}$.

\section{Interprétations ritualistes}

Empruntée à l'anthropologie comparée, la notion de "rite d'initiation tribale" (destiné aux adolescents), qui s'inscrit dans la catégorie plus générale des "rites de passage", ne va pas tarder à donner à la lecture moderne des récits hellènes une impulsion nouvelle. Avec ses trois phases de séparation de l'ordre ancien, de période de marge correspondant à une mort symbolique et de réintégration avec le statut nouveau acquis par la ou le néo-initié, le schéma du rite de passage - on l'aura compris - offre une clé de lecture rêvée dans l'interprétation de l'enlèvement de Coré, de sa disparition aux Enfers et de son retour auprès de sa mère. Il permet en effet non seulement de lire le "mythe" dans la dynamique de son déroulement narratif, mais il induit également l'interprète à intégrer et à combiner dans un même paradigme herméneutique données rituelles et manifestations discursives.

On doit à Henri Jeanmaire l'insertion de l'histoire du rapt de Perséphone dans la catégorie dynamique du rite de passage. Inscrite dans un nom propre évoquant celui de la "jeune fille" (kóre), la jeunesse même de Coré insère son histoire dans la classe spécifique des rites de passage que constituent "les rites d'adolescence" étudiés par l'historien français 
de la religion grecque. Mais pour que réussisse cet "essai d’interprétation", le récit de la "descente et remontée de Coré enlevée par le Seigneur du Monde infernal et reconquise par la douleur et l'amour de sa mère" est mise en relation non pas avec les Mystères d'Eleusis, mais avec les Thesmophories. C'est en effet à ce festival panhellénique que Jeanmaire attribue le rituel athénien de la consécration des plásmata faits d'argile et des restes putréfiés des porcelets jetés au préalable dans de mystérieuses anfractuosités du sol. Même si elle est jugée un peu "fruste" et schématique, une première homologie est tracée entre les "avatars successifs du rapt et de la délivrance d'une déesse" et cet "acte religieux vulgaire et relativement simple de précipiter une victime en pâture aux génies du monde souterrain et d'en utiliser les restes pour la confection d'un talisman de fertilité"15. Le mythe représenterait ainsi l'idéalisation d'un rituel plutôt macabre.

Réinterprété à l'aide du rite en termes de "descente" et de "remontée" (káthodos et ánodos), le déroulement du parcours de Coré devrait lui-même refléter un rite de passage qui subsisterait ainsi dans le mythe à l'état de "survivance". Dès lors l'Hymne homérique à Déméter ne ferait que "narrer le vieux mythe à la façon d'une sorte de roman bourgeois", sinon de "conte de fée". Quoi qu'il en soit de la forme romancée assumée par le récit, la légende de Perséphone comme celle du voyage de Thésée en Crète reprendraient le schéma du rite de passage destiné aux adolescents. Grâce à la notion de survivance qui restitue à l'interprétation une dimension historique de type évolutionniste, le rite de passage destiné aux adolescents pour assurer leur accession à l'âge adulte est transformé en clé de lecture non seulement des rituels de l'âge classique, mais aussi de récits présentant soit un moment de rupture (le rapt), soit une figure adolescente (la jeune Coré). A ce jeu-là, l'articulation triadique et dynamique du schéma rituel avec l'enchaînement nécessaire de ses trois phases constitutives n'est plus au centre de la préoccupation de l'herméneute lecteur de textes.

Mais c'est des Etats-Unis qu'est venu l'essai le plus conséquent de voir dans l'histoire de l'enlèvement de Perséphone par Hadès et de son retour en raison des plaintes de sa mère le développement d'un rite d'initiation destiné à faire passer la jeune fille à l'état de femme adulte. Située au printemps, la disparition de Coré ne correspond ni à l'enfouissement du grain dans le sol (et donc au début de la saison de l'hiver ou de l'été!) comme le voudraient les tenants de l'interprétation allégorique du mythe, ni au début d'une cérémonie de mariage puisque l'époux, Hadès, est à la fois l'oncle maternel et paternel de la "fiancée". Mais le séjour dans les profondeurs des Enfers coïncide avec le lieu et la période de séclusion d'un rite d'initiation qui consacre pour la jeune fille, avec la perte violente de sa virginité, la transition à l'âge adulte dans le "betwixt and between" d'une période liminale ${ }^{16}$. A l'occasion de ce passage, Coré, "the maiden", perd son identité générique pour acquérir, en tant que Perséphone, l’identité individuelle que confère la dation d'un nom propre. Par comparaison, la tentative d'immortalisation de Démophoon peut alors elle-même apparaître comme l'échec d'une initiation à l'enfance. La lecture initiatique du destin de Coré reporte donc l'interprétation du cycle du renouveau de la végétation vers l'action rituelle et sociale des hommes.

Il est évident qu’à projeter trop systématiquement sur le récit un schéma rendant compte de pratiques rituelles, on oublie non seulement qu'au terme du processus "initiatique" que suivrait sa biographies, Perséphone reste pour les deux tiers de l'année au moins la fille de sa mère, mais aussi qu'elle n'acquiert jamais le statut de la femme accomplie signifié en Grèce par la 
naissance du premier enfant. Auprès d'Hadès elle est et reste une númphe, une jeune mariée, sans jamais devenir une guné. La consommation même du fameux pépin de grenade destiné à retenir la jeune femme aux Enfers pendant le tiers restant de l'année montre que ce séjour temporaire est constamment marqué par la puissance de la déesse Aphrodite.

\section{Vers la psychologie des profondeurs}

Mais on sait fort bien que, pas plus en herméneutique que dans d'autres domaines des sciences humaines ou dures, l'histoire ne suit un cours ni progressif, ni linéaire. Au moment où Jeanmaire élaborait une interprétation initiatique des récits de Perséphone, Karl ou Carl Kenényi découvrait chez les Wemale de Ceram dans les Moluques, par l'intermédiaire des travaux de l'historien des religions et anthropologue Adolf E. Jensen, des figures et des pratiques rituelles évoquant à ses yeux "le mythe de la religion éleusinienne". Si la notion d'initiation soutient son analyse des gestes rituels formant le noyau des Mystères, son interprétation de leurs actes dédicatoires marque un retour aux catégories statiques des figures abstraites. En tant que "figure primordiale", Coré devient l'incarnation grecque de la Divine Maiden, à côté de la figure du Divine Child, comprise comme appartenant au "thème mythologique" du Primordial Child. Quant à Déméter, elle est appelée à représenter à la fois la fondatrice des Mystères et la première initiée puisque la recherche de sa fille est comprise comme une initiation. Mais, par les jeux de mots déjà largement exploités, Déméter devient également une figure particulière de Terre; elle serait la mère du grain s'il est bien vrai qu'en mycénien la syllabe da désigne une mesure permettant d'arpenter les champs de blé ${ }^{17}$.

C'est alors dans le dialogue avec Carl G. Jung que les figures primordiales de l'Enfant (Primordial) et de la Jeune Fille divine se transforment en "archétypes" transitant ainsi du domaine de l'histoire de la religion universelle dans celui d'une psychologie des profondeurs. Dès lors, sur la base des figures de Coré-Perséphone et de Déméter, la Mère (en tant que Mère Primordiale et Mère-Terre) et la Fille prennent place chez Jung parmi les autres archétypes que sont l'Ombre, le Vieillard de sagesse, le Soi ou l'Enfant; ces figures constituent les archétypes se combinant avec Anima chez l'homme et Animus chez la femme pour fonder le fonctionnement de la psyché et de l'inconscient. Dans cette perspective, le "mythe" de Déméter et Coré, compris comme la relation archétypale entre mère et fille, n'est pas uniquement le résultat d'une projection de l'Anima en tant qu'image masculine idéalisée de la féminité; il est aussi l'expression d'une expérience dont l'homme est par définition exclu, une expérience renvoyant à un "ordre matriarcal de la société". Si pour Kerényi la relation Mère-Fille est constitutive de la vie psychique de chaque être humain, femme et homme, chacun à sa manière, selon Jung c'est avant tout la destinée de la femme qui est marquée par la constante tension temporelle entre l'expérience de la fille et celle de la mère; à travers mythes et rêves, l'inconscient féminin est animé par les archétypes que représentent Perséphone et Déméter.

La tentative herméneutique conjointe de Jung et Kerényi signifie donc non seulement un retour à des catégories et à des concepts dont les figures divines ne sont que les représentations dans un mythe grec transformé en substance; mais elle correspond aussi au postulat d'un état originaire qui est situé autant dans le temps de l'histoire que dans celui de 
la psyché. L'ordre matriarcal que l'on suppose à l'origine de la société est ainsi projeté dans l'histoire de la vie psychologique profonde de l'individu, essentiellement dans celle de la femme. Comme c'est le cas en particulier pour le complexe d'Edipe, mais de manière beaucoup plus statique, le récit grec, réduit à ce qui n'est même plus une intrigue, est non pas interprété, mais réutilisé pour figurer le fonctionnement supposé du psychisme humain.

L'usage de la relation entre Déméter et Coré proposé par Jung et Kerényi n'a évidemment pas échappé aux lectrices américaines de l'Hymne homérique à Déméter, engagées à différents degrés dans la légitime promotion du gender entend comme représentation des relations sociales de sexe. Les "Women Studies" ne pouvaient donc pas rester indifférentes à une relation entre mère et fille essentiellement saisie dans sa composante émotionnelle. On ne fera que mentionner les essais récents de faire des différents épisodes de l'histoire de l'enlèvement de la fille et de sa recherche par la mère des emblèmes rerpésentant les stades successifs du développement psycho-sexuel de la femme: régression au stade phallique de la fille découvrant son manque de pénis et identification avec sa mère dans son amour pour le père à travers la crise que provoquent chez Déméter la manifestation d'autorité de Zeus (par l'intervention brutale d'Hadès) et le passage forcé du matriarcat à une société patriarcale; roman de Coré et de Déméter comme figuration du développement psycho-affectif de l'enfant vivant originairement en symbiose avec sa mère et subissant la première crise œdipale dans l'acquisition d'une identité de genre à l'intérieur d'un système patriarcal ${ }^{18}$.

Faire des avatars de la relation entre Coré et Déméter la représentation soit du développement inconscient de la psyché de la jeune fille, soit de son évolution psychologique au moment de l'adolescence, c'est placer deux déesses grecques, protagonistes d'un récit à fonction étiologique et cultuelle, sur le divan du psychanalyste ou dans le fauteuil du thérapeute. C'est parce que l'effet de réel de la fiction narrative est pris au pied de la lettre que les deux figures divines peuvent devenir des patientes dont le cas est promu au rang de modèle; il devient la représentation figurée d'un fonctionnement psychologique ou psychanalytique implicitement donné comme universel. Il s'agit en fait, dans une procédure interprétative devenue constitutive et par l'effet du biais féministe, d'un nouveau phénomène de naturalisation: le développement de la nature humaine - dans le cas particulier celle de la femme a remplacé le cycle de la nature végétale.

\section{Perspectives critiques}

Que ce soit par l'intermédiaire de l'étymologie, par la référence aux grands cycles de la nature (sinon du cosmos), par la comparaison avec le schéma du déroulement d'un rituel (initiation tribale ou sacrifice), par la réduction au fonctionnement supposé de la psyché ou par la construction d'oppositions binaires articulées en différents codes, le déroulement du récit réduit à sa trame mythographique ou réalisé dans un poème tel l'Hymne homérique à Déméter est ramené à quelques entités abstraites organisées en une relation plus ou moins dynamique. Dans son mouvement historique, cette procédure herméneutique repose en définitive sur un principe analogue à celui qui a conduit à la notion moderne de mythe. Elle revient également à la création de catégories du "niveau de base", entités qui partagent leur 
réalité entre l'empirique et le figuratif d'une part, le notionnel et l'abstrait de l'autre; les procédures de la métaphore et de la métonymie jouent, dans leur construction et pour leur établissement dans une tradition en général érudite, un rôle essentiel, assurant notamment le passage constant de l'humain au minéral et au végétal.

Il ne fait aucun doute que ces catégories semi-empiriques, en tant qu'outils herméneutiques, réalisent pleinement leur fonction de création d'un cadre de référence en accord avec une certaine communauté de croyance (en général académique), en accord aussi avec un paradigme intellectuel donné. Elles représentent assurément des instruments de connaissance susceptibles d'insérer des récits que la distance temporelle et spatiale rend de plus en plus étrangers dans ce que l'on appelera de nouveaux "régimes d'intelligibilité". Cette procédure d'ordre interprétatif, qui fait des catégories "du niveau de base" les instruments d'une sorte de traduction transculturelle, est d'autant plus frappante qu'elle est à l'oeuvre dès l'Antiquité, preuve que les récits spéculatifs que nous appelons mythes suscitent euxmêmes réflexion, spéculation et finalement connaissance. L'aspect transculturel de la procédure interprétative exposée rend illusoire la fixation de l'un ou l'autre des cadres de référence qui la fondent; leur stabilisation n'est jamais que provisoire. Chaque "mythe" ne peut être lu que dans la version particulière qui le porte à son destinataire ${ }^{19}$.

Ce n'est donc guère que dans un texte poétique achevé tel que l'Hymne homérique à Déméter que l'on pourra appréhender, autant dans sa syntaxe que dans ses valeurs propres et dans son aspect pragmatique, la manifestation discursive toujours singulière se donnant à nous sous les aspects d'un récit dit mythique. Ce n'est qu'au prix d'une ascèse appelée à éviter les abstractions trop rapides et réductrices que l'on verra que, dans le poème homérique par exemple, le récit du rapt et du retour de Perséphone est d'une part inséparable de la tentative d'immortalisation de Démophoon et que sa narration est d'autre part liée de manière constitutive à la célébration du culte rendu aux deux déesses, à Eleusis. Les récits en général poétiques que l'on appréhende depuis la fin du XVIII ${ }^{e}$ siècle par l'appellation et la notion de "mythe" ne se réduisent certainement pas à de simples manières de dire une réalité plus abstraite ou plus fondamentale. La trame narrative du rapt de Perséphone telle qu'elle est réalisée dans l'Hymne homérique adressé aux deux déesses ne peut être comprise que dans l'articulation complexe des effets de sens qu'elle provoque et dans la relation pratique du poème avec le déroulement et l'accomplissement du rituel que le récit est censé instituer. Syntaxe, assurément, mais aussi sémantique et pragmatique pour des récits faisant référence, dans chacune de leurs "performances", à des représentations et à des pratiques culturelles et symboliques très développées de la vie en société.

\section{Un récit en acte: I'Hymne homérique à Déméter}

Au-delà donc des interprétations indigènes ou modernes, seule l'étude du récit dans l'une de ses manifestations génériques, en portant notamment l'attention sur les marques de sa mise en discours, est susceptible de restituer les valeurs peu à peu construites dans le développement de la narration fictionnelle. L'Hymne homérique à Déméter est une composition poétique d'autant plus intéressante qu'elle représente, par sa référence et son adresse à la divinité, un proème cultuel à la récitation rituelle d'un poème épique. Dans la perspec- 
tive de la logique et de la sémantique du récit raconté dans cet hymne, lui-même en diction épique, on relèvera en particulier le statut intermédiaire gagné par Perséphone dans la phase de sanction de la narration, de par la volonté conjuguée de Zeus et de son frère Hadès: à la fois fille de sa mère sur l'Olympe et épouse (mais dans un mariage sans enfant) du maître des Enfers. Cet état mixte, qui ne saurait correspondre à celui atteint à la suite d'un rituel d'initiation tribale, doit être mis en relation, du point de vue narratif, avec l'institution d'un premier rituel à mystère par Déméter elle-même; du point de vue énonciatif, il coïncide avec la double formule de macarismos qui précède immédiatement la conclusion de l'hymne. L'incorporation au récit lui-même de cette démonstration par la divinité en personne des órgia kalá, rites respectables qu'il est d'emblée interdit de divulguer ou de transgresser et dont la célébration est confiée aux maîtres d'Eleusis représente non seulement la deuxième phase de sanction de ce récit; mais, en combinaison avec la formule d'adresse indirecte aux mortels bienheureux qui conduit la composition à sa conclusion, ce dernier acte narratif de la déesse s'inscrit dans la perspective étiologique qui traverse tout le poème.

Le récit hymnique du rapt de Coré par Hadès de la surface terrestre, du séjour de sa mère auprès des souverains mortels d'Eleusis, puis du retour partiel de la jeune épouse sur l'Olympe est en effet ponctué d'épisodes qui évoquent les gestes rituels accomplis dans la célébration cultuelle instituée par Déméter elle-même; ces actes de culte constitueront les Mystères d'Eleusis dès qu'Athènes aura pris le contrôle politique et religieux de la cité voisine. C'est le cas par exemple de l'abstinence à l'égard du nectar et de l'ambroisie que Déméter s'impose dans sa colère et qui rappelle le jeûne suivi par les futurs initiés de même que les torches tenues par la déesse dans la quête de sa fille évoquent les flambeaux éclairant rituellement la cérémonie de l'initiation elle-même. Il est légitime de mettre également en relation le rôle de nourrice que la mère de Coré assume vis-à-vis de Démophoon dans sa tentative d'immortaliser le jeune enfant avec la fonction équivalente attribuée aux deux déesses à l'égard des initiants, les railleries et gestes obscènes de Iambé avec les injures rituelles qui ponctuaient la procession vers Eleusis, ou la préparation de la mixture de farine d'orge, d'eau et de menthe offerte à Déméter sur ses propres indications avec le breuvage qui deviendra la boisson rituelle des néo-initiés. Ainsi en va-t-il a fortiori de tous les gestes explicites d'institution ou de fondation qui marquent la deuxième partie de la composition hymnique: construction d'un temple et d'un autel consacrés à Déméter au pied de l'acropole d'Eleusis; institution par la déesse elle-même des órgia, sacrifices et offrandes promis à Perséphone pendant son séjour dans l'Hadès où elle régnera sur tous les êtres mortels; transformation d'Hécate en suivante de Coré; enseignement par Déméter des procédures rituelles à suivre dans la célébration des órgia ${ }^{20}$.

La narration du séjour infernal de Perséphone telle qu'elle est conduite dans l'Hymne homérique trouve donc sa dimension pragmatique dans la relation étiologique que le récit établit de manière constante avec le rituel auquel est destiné son exécution. Il y a en effet tout lieu de croire qu'en relation avec la célébration des Mystères, la composition hymnique dont nous avons le texte était chantée à l'occasion des jeux d'Eleusis. Attestés pour nous dès la fin du VI ${ }^{e}$ siècle av. J.-C., ces jeux incluaient certainement un concours d'aèdes ou de rhapsodes; les récitations épiques de ces récitants homériques étaient introduites par les offrandes chantées aux dieux que représentent les compositions poétiques et narratives assumant la forme de l'Hymne homéri$q u e^{21}$. Dans ce contexte rituel, et par le passage intradiscursif du récit à l'énoncé de l'énonciation, 
le double macarismos qui conclut le poème offre une double adresse du locuteur aux initiés en général; par la promesse d'une destinée meilleure dans leur vie sur et sous terre, il renforce la valeur performative du poème comme acte de culte. Par différentes stratégies énonciatives conduites par le locuteur-narrateur, non seulement l'hymne est offert à la puissante et généreuse Déméter ainsi qu'à sa fille Perséphone en échange d'une vie prospère accordée à un poète qui inscrit son chant dans la logique du do ut des propre à la prière cultuelle; mais le poème proclame également le bonheur et la prospérité des mortels qui ont accompli les pratiques rituelles instituées par Déméter elle-même.

A l'égard de toutes celles et ceux qui n'ont vu dans le récit de Déméter et de Coré que le drame de la végétation mourante puis renaissante, l'histoire de l'institution pour les humains de la civilisation céréalicole ou le destin psychique de la fille à la recherche de son identité sexuelle, on pourra relever, au moins en ce qui concerne la version activée dans le poème hymnique, la remarquable correspondance entre le statut très singulier que partage finalement Perséphone entre Olympe et monde infernal, et le destin de l'homme mortel; par le rituel initiatique, celui-ci peut souhaiter, un peu comme un dieu, prolonger sa vie sur terre, puis connaître après son trépas un sort plus heureux aux Enfers. Du point de vue de l'espace dessiné dans le récit, la position intermédiaire des mortels est signifiée par le double séjour de Perséphone, dans le ciel et aux Enfers après qu'elle a été enlevée de la surface terrestre. Condamnés par leur mortalité à séjourner sur terre, les humains ne peuvent qu'espérer obtenir par les moyens de l'acte de culte une vie dans l'abondance qui les rapprocherait du monde divin et une mort qui ne les condamnerait pas à l'inconsistance entière des défunts.

Au-delà du processus de spatialisation du récit, cette correspondance métaphorique entre le statut divin construit dans la narration fictionnelle et la destinée rituelle des initiés est inscrite dans sa temporalité. La phase de sanction du récit qui décrit, à l'aoriste, la révélation par Déméter des pratiques rituelles à accomplir dans la célébration des futurs Mystères débouche sur un premier macarismos annonçant au présent le bonheur obtenu par celui qui les a vus. Le retour des deux vénérables déesses sur l'Olympe qui marque, de nouveau à l'aoriste, la fin de la phase de sanction conduit non seulement au séjour permanent des deux divinités auprès de Zeus, mais aussi au second macarismos qui vante, au présent, le bonheur et la richesse provoqués pour les mortels et leur maisonnée par l'amour que peuvent leur porter Déméter et Coré. Cette seconde adresse au genre humain en général débouche sur la partie performative à proprement parler du poème: par le moyen des formes impératives ou intentionnelles du futur proche, le locuteur-narrateur offre son propre poème en échange de la prospérité demandée aux deux déesses. Ainsi, grâce aux traces énonciatives laissées par une mise en discours qui subordonne toute la narration aux adresses et aux voeux concluant le poème, le chant devient prière; il devient acte de culte.

La sanction du récit confirme donc la position des mortels, sexes confondus, dans l'entredeux; non pas entre dieux et bêtes (celles-ci sont, sinon sous la forme des êtres vivants dont les humains font partie, totalement absentes du récit), mais entre monde d'en-haut et monde d'enbas, entre divinités olympiennes sous le contrôle de Zeus le père et âmes des défunts placées sous le pouvoir de son frère Hadès. Quand ils sont favorisés par Déméter la nurse et la nourricière, le travail et la production agricoles comme les Mystères ne peuvent qu'accroître la prospérité d'humains de toute façon destinés à la mortalité, sans différence de sexe. 
Ainsi, notamment par un double et subtil processus de spatialisation et de temporalisation narratives, la fiction du rapt de Coré sur une prairie de séduction, et de l'établissement de Perséphone comme jeune épouse encore sans descendance entre son oncle dans l'Hadès et sa mère (et son père) sur l'Olympe, avec le parcours de type initiatique qu'implique le récit, renvoie à une importante configuration idéologique; dans la représentation grecque classique et par métaphore interposée, cette constante idéologiqueassocie le processus social du mariage à la production céréalière ainsi qu'à un premier état de civilisation $^{22}$. Mais autant en raison des valeurs intermédiaires que le récit attribue au statut final de la jeune Perséphone au terme de son itininéraire que par les stratégies énonciatives que développe le discours fictionnel en suivant les règles génériques de l'hymne de type homérique avec sa conclusion performative, la narration du parcours de Coré trouve sa raison d'être dans la pratique rituelle, puis dans la conduite sociale visant à obtenir aisance matérielle ici-bas et survie dans l'au-delà. La performance narrative et hymnique, présentée dans l'adresse finale comme une offrande aux deux divinités, doit y contribuer, pour le bénéfice des hommes en général, et pour celui du locuteur et aède en particuler. La construction fictionnelle et symbolique, par les moyens des potentialités de la langue et des règles du genre, réalise ainsi pleinement sa dimension pragmatique, sinon cognitive. Le récit du rapt de Perséphone tel qu'il est orienté par l'hymne aédique se présente comme un chant louant et effectuant dans le rituel le "Greek way of life".

\section{En guise de coda}

Il conviendra de rappeler en conclusion et en relation avec le thème de la rencontre de Diamantina, que les Mystères d'Eleusis n'étaient pas uniquement composés de legómena et de drómena qui pouvaient correspondre à l'Hymne homérique lui-même et aux pratiques rituelles dont le récit poétique chante l'institution. Mais s'y ajoutaient les deiknúmena qui se trouvaient au centre de la célébration et de la révélation rituelle des Mystères. Pour reprendre les réflexions présentées en guise d'apéritif par l'organisateur du colloque, il faut aussi se souvenir que les Grecs ont toujours été soucieux des capacités de la parole, qu'ils ont tant cultivée, à faire voir. Sans doute n'est-ce pas un hasard si l'adjectif enárges, qui qualifie dans la poésie homérique la luminosité de l'épiphanie d'une divinité, se retrouve dans le substantif enárgeia pour désigner dans la rhétorique classique la qualité du discours qui parvient à "présenter au regard", et par conséquent à solliciter la vue de l'auditeur. Voix et vue ont pour les Grecs (et les Romains) des affinités que l'Hymne homérique à Déméter, dans sa dimension performative, ne cesse de valoriser.

\section{Notes}

1 - L'impact de l'institution de la mythographie à partir de Platon a été indiqué par Detienne, 1981, p. 160-7. Sur la mythographie elle-même, voir Henrichs, 1987, Pellizer, 1993, et Jacob, 1994. Quant à la définition du mythe grec comme "récit traditionnel", voir, à partir d'une proposition formulée par W. Burkert, les précisions apportées par Bremmer, 1997. 
2 - D’Alembert et Diderot, 1757, VIII, p. 98; voir aussi, par exemple, VI, p. 342, où la figure d'Hélène est en revanche classée sous la rubrique Histoire ancienne! Sur cette histoire de la notion de "mythe" à partir du Siècle des Lumières en contraste avec les sens de mûthos en Grèce même, on verra les indications que j'ai données dans l'ouvrage de 1996, p. 8-46

3 -Lévi-Strauss, 1964, p. 14-22; voir également les remarques qui concluent l'entreprise de 1971, p. $559-621$.

4 - Avec une focalisation sur la Grèce ancienne, cette histoire de l'interprétation des mythes a été récemment et successivement retracée par Detienne, 1981, p. 15-49 et 190-224, Graf, 1993, p. 9-56, Saïd, 1993, p. 79-110, études auxquelles on ajoutera les réflexions de Vernant, 1974, p. 217-50, ainsi que l'étude de Burkert, 1980, et l'ouvrage de Jamme, 1991.

5 -Ps. Apollod. Bibl. 1, 5, 1-3. Tout juste mentionné dans l'HCer. 153 comme l'un des "souverains d'Eleusis", Triptolème joue un rôle central dans les versions traitées par les Atthidographes (Pherec. Ath. FGrHist. 3 F 53, Philoch. FGrHist. 328 F 104, etc.), par les grands tragiques (Soph. frr. 596-617a Radt), ou par les imagiers de la céramique attique dès 540 environ. Les différentes versions du "mythe" sont analysées par Richardson, 1974, p. 74-86 (sur Triptolème, voir aussi Foley, 1994, p. 194-8), et par Foley, 1994, p. 97-103; pour l'iconographie, voir en particulier Shapiro, 1989, p. 67-83.

6 - Isocr. Paneg. 26-33.

7 - Cic. Nat. deorum 2, 66-7; à propos de l'interprétation allégorique proposée par les Stoïciens du rapt de Perséphone on trouvera d'autres références encore chez Richardson, 1974, p. 284. Sur le rôle des jeux de mots étymologisants dans l'allégorie stoïcienne, voir Pépin, 1976, p. 12431. Dans l'un des célèbres textes d'oracle delphique énoncés à propos de la prise d'Athènes par les Perses et cités par Hérodote $(7,141,4)$, c'est Déméter qui représente le blé.

8 -Lafitau, 1724, I, p. II-III et 220-40; voir à ce propos Kilani, 1994, p. 77-103.

9-Vico, 1744, I, p. 145-51, 266-7 et 331-3 (pagination de l'édition de F. Nicolini, Bari: Laterza, 1928); sur le rapt de Perséphone: cf. p. 244-5, 348 (ainsi que 242 et 350 pour l'assimilation de Coré avec Déméter) et 240-1. Voir Calame, 1996, p. 14-5.

10 - Muller, 1881, p. 403-31 et 536-7 (cf. Hdt. 2, 52 et Plat. Crat. 397c), avec les indications bibliographiques et les remarques formulées à ce sujet par Burkert, 1980, p. 165-8.

11 - Frazer, 1913, p. 35-91.

12 - Frazer, 1913, p. 91.

13 - Nilsson, 1925/1952, se référant notamment pour la katagogé de Coré à Diod. Sic. 5, 4, 5-6; voir aussi les indications bibliographiques complémentaires données à ce propos par Richardson, 1974 , p. 284-5, ainsi que Burket, 1972, p. 286-92.

14 - Richardson, 1974, p. 13-4. A propos des difficultés que l'on rencontre à accorder le temps du récit du rapt de Coré avec la chronologie festive du calendrier et le cycle des travaux agricoles, voir encore mon étude de 1997.

15 -Jeanmaire, 1939, p. 264-312. Sur le rituel du jet des porcelets, cf. Burkert, 1972, p. 284-67.

16 - Lincoln, 1979.

17 - Kerényi fait l'histoire de sa découverte et de son dialogue avec C. Jung dans l'ouvrage de 1960, p. XXIII-XXXVII; voir aussi p. 23-9 ainsi que p. 130-1 où, par analogie avec la Mère grain mother, la Fille devient grain maiden; le premier état de la recherche comparative conduite par Kerényi a été publié en particulier dans l'article de 1940.

18 -Cf. Arthur, 1973, et Foley, 1994, p. 118-37, se fondant sur l'étude de Chodorow, 1974; les deux études d'Arthur et de Chodorow ont été reprises par Foley, 1994, p. 214-42 et p. 243-65 respectivement.

19 - Voir les différentes contributions publiées dans Calame (ed.), 1988. 
20 - On a cité successivement les vers 470-89, 47-50, 231-45, 201-3, 207-11, 268-74 (cf. aussi 296-302), 364-9, 438-40 et 473-9 de l'HCer; on lira au sujet de la fonction étiologique de tous ces passages le commentaire circonstancié qu'en a donné Richardson, 1974, sans oublier les p. 326-30 et 344-8; voir aussi Konstan, 1996. On trouvera quelques indications allant dans la direction de la lecture proposée ici de la version "homérique" du rapt de Coré dans mon article de 1997, et une série de références bibliographiques sur l'Hymne homérique dans sa fonction cultuelle de proème dans mon étude de 1995.

21 - Voir à ce propos Richardson, 1974, p. 5-12 et 20-30, qui cite notamment $I G$ I2, 5; pour le concours analogue qui marquait la célébration des Panathénées, lire en dernier lieu Shapiro, 1992.

22 - En particulier les études de Rudhardt, 1978, et de Strauss Clay, 1987, p. 202-66, sont sensibles au dessin spatial de cette composition hymnique.

NB: La présente étude est extraite de la version provisoire du chapitre introductif d'un ouvrage consacré à la Poétique des mythes dans la Grèce antique, désormais paru à Paris: Hachette, 2001, p. 11-69. J'aimerais exprimer ma reconnaissance aux organisateurs du colloque de Diamantina, en partie sous l'égide du PARSA, pour m'avoir permis, grâce à leur généreuse hospitalité, de soumettre à la critique les présentes réflexions.

\section{Références bibliographiques}

ARTHUR, M. Politics and pomegranates: an interpretation of the Homeric Hymn to Demeter. In: Arethusa 6, 1973, p. 7-47.

BREMMER, J. What is a Greek myth? In: BREMMER, J. (ed.). Interpretations of Greek mythology. London/ Sydney: Croom Helm, 1987, p. 1-8.

BURKERT, W. Griechische Mythologie und die Geistesgeschichte der Moderne. In: REVERDIN, O. (ed.). Les études classiques aux XIXe et XXe siècles (Entretiens Hardt XXVI). Vandoeuvres/Genève: Fondation Hardt, 1980, p. 159-99.

Homo Necans. Interpretationen altgriechischer Opferriten und Mythen, Berlin/New York: de Gruyter, 1972.

CALAME. C. Variations énonciatives, relations avec les dieux et fonctions poétiques dans les Hymnes homériques. In: Mus. Helv. 52, 1995, p. 2-19.

Mythe et histoire dans l'Antiquité grecque. La création symbolique d'une colonie. Lausanne: Payot, 1996.

L'Hymne homérique à Déméter comme offrande: regard rétrospectif sur quelques catégories de l'anthropologie de la religion grecque. In: Kernos 10, 1997, p. 111-33.

CALAME. C. (ed.). Métamorphoses du mythe en Grèce antique. Genève: Labor \& Fides, 1988.

CHOROROW, N. Family structure and feminine personality. In ROSALDO, M. Z., LAMPHERE, L. (edd.). Woman, Culture and Society. Stanford: Stanford University Press, 1974, p. 43-66.

D'ALEMBERT, J. \& DIDEROT, D. Encyclopédie ou dictionnaire raisonné des sciences, des arts et des métiers. VIII. Paris: Didot, 1757. 
DETIENNE, M. L'Invention de la mythologie. Paris: Gallimard, 1981.

FOLEY, H. P. The Homeric Hymn to Demeter. Princeton: Princeton University Press, 1994.

FRAZER, J. G. The golden bough. A study in magic and religion. Part V: Spirits of the Corn and of the Wild I. London: Macmillan, 1913.

GRAF, J. Greek mythology. An introduction. Baltimore/London: The Johns Hopkins University Press, 1993.

HENRICHS, A. Three approaches to Greek mythography. In: BREMMER, J. (ed.), Interpretations of Greek mythology. London, Sydney: Croom Helm, 1987, p. 242-77.

JACOB, Ch. L'ordre généalogique. Entre le mythe et l'histoire. In: DETIENNE, M. (ed.). Transcrire les mythologies. Tradition, écriture, historicité. Paris: Albin Michel, 1994, p. 169-202.

JAMME, Ch. Einführung in die Philosophie des Mythos II. Neuzeit und Gegenwart. Darmstadt: Wissenschaftliche Buchgesellschaft, 1991.

JEANMAIRE, H. Couroi et courètes. Essai sur l'éducation spartiate et sur les rites d'adolescence dans l'antiquité hellénique. Lille: Université, 1939.

KERENYI, K. Kore: zum Mythologem vom Göttlichen Mädchen. In: Paideuma 1, 1940, p. 341-80.

Eleusis. Archetypal image of mother and daughter. New York: Schocken, 1967 (ed. or. holl. The Hague, 1960)

KILANI, M. L'invention de l'autre. Essais sur le discours anthropologique. Lausanne: Payot, 1994.

KONSTAN, D. De Deméter a Ceres: construcciones de la diosa en Homero, Calímaco y Ovidio. In: Synthesis 3, 1996, p. 67-90.

LAFITAU, J.-F. Moeurs des sauvages amériquains comparées aux moeurs des premiers temps. Paris: Noury, 1724.

LEVI-STRAUSS, C. Mythologiques I. Le cru et le cuit. Paris: Plon, 1964. . Mythologiques IV. L'homme nu. Paris: Plon, 1971.

LINCOLN, B. The rape of Persephone: a Greek scenario of women's initiation. In: Harv. Theol. Rev. 72, 1979, p. 223-35.

MUELLER, (F.) M. Lectures on the science of language. Second Series. London: Longmans Green, 1881.

NILSSON, M. P. Die eleusinischen Gottheiten. In: Arch. Religionswiss. 32, 1925, p. 79-141 (repris in: Opuscula Selecta II, Lund: Gleerup, 1952, p. 542-613).

PELLIZER, E. La mitografia. In: CAMBIANO, G., CANFORA, L., LANZA, D. (edd.). Lo spazio letterario nella Grecia antica II. Roma: Salerno, 1993, p. 283-303.

PEPIN, J. Mythe et allégorie. Les origines grecques et les contestations judéo-chrétiennes. Paris: Etudes Augustiniennes, 1976 (2e éd.).

RICHARDSON, N. J. The homeric hymn to Demeter. Oxford: Clarendon Press, 1974.

RUDHARDT, J. A propos de l'hymne homérique à Déméter. In: Mus. Helv. 35, 1978, p. 1-17. 
SAID, S. Approches de la mythologie grecque. Paris: Nathan, 1993.

SHAPIRO, H. A. Art and cult under the tyrants in Athens. Mainz: von Zabern, 1989. Mousikoi agones: music and poetry at the panathenaia. In: NEILS, J. (ed.). Goddess and polis. The panathenaic festival in ancient Athens. Princeton: Princeton University Press, 1992, p. 53-75.

STRAUSS CLAY, J. The politics of Olympus. Form and meaning in the major homeric hymns. Princeton: Princeton University Press, 1987.

VERNANT, J.-P. Mythe et société en Grèce ancienne. Paris: Maspero 1974.

VICO, G. La scienza nuova. Napoli: Mosca, 1744 ( $2^{e}$ éd.).

CALAME, Claude. Le récit du rapt de Perséphone: interprétations d'un "mythe" et performance rituelle. Classica, São Paulo, 13/14, p. 17-35, 2000-2001.

RÉSUMÉ: Le récit du rapt de Perséphone fournit un cas de figure idéal pour introduire à la fois à une critique de la notion moderne de "mythe" et à un parcours à travers les différentes interprétations que ce récit constitué en "mythe" a pu susciter. Seule l'étude d'une version poétique particulière, replacée dans ses circonstances d'énonciation rituelles par l'intermédiaire d'une approche à la fois anthropologique et sémio-narrative, est susceptible de rendre compte des valeurs et de la fonction propres d'un "mythe".

MOTS-CLÉS: Poétique; mythe grec; Déméter et Perséphone; herméneutique. 